\title{
Correction: Phylogenetic, genomic, and biogeographic characterization of a novel and ubiquitous marine invertebrate- associated Rickettsiales parasite, Candidatus Aquarickettsia rohweri, gen. nov., sp. nov
}

\section{J. Grace Klinges ${ }^{1} \cdot$ Stephanie M. Rosales ${ }^{1,2,3} \cdot$ Ryan McMinds $^{1,4} \cdot$ Elizabeth C. Shaver $^{5} \cdot$ Andrew A. Shantz $\mathbb{D}^{6,7}$. Esther C. Peters $\mathbb{1}^{8} \cdot$ Michael Eitel $^{9} \cdot$ Gert Wörheide $\mathbb{1}^{9,10,11} \cdot$ Koty H. Sharp ${ }^{12} \cdot$ Deron E. Burkepile ${ }^{6}$.}

Brian R. Silliman ${ }^{5} \cdot$ Rebecca L. Vega Thurber $^{1}$

Published online: 24 September 2019

(c) The Author(s) 2019

\section{Correction to: The ISME Journal}

$$
\text { https://doi.org/10.1038/s41396-019-0482-0 }
$$

Since publication of the original article the authors noticed that an incorrect file was uploaded instead of the
Supplementary Tables. The incorrect file has been replaced with the Supplementary Tables file. Furthermore the abstract has been edited slightly in both HTML and PDF versions for clarity.
Rebecca L. Vega Thurber

rvegathurber@gmail.com

Rebecca.Vega-Thurber@oregonstate.edu

1 Department of Microbiology, Oregon State University, Corvallis, OR 97331, USA

2 Cooperative Institute for Marine and Atmospheric Studies, University of Miami, Miami, FL 33149, USA

3 Atlantic Oceanographic and Meteorological Laboratory, National Oceanographic and Atmospheric Administration, Miami, FL 33149, USA

4 Center of Modeling, Simulation and Interactions, Université Côte d'Azur, Nice, France

5 Division of Marine Science and Conservation, Nicholas School of the Environment, Duke University, Beaufort, NC 28516, USA

6 Department of Ecology, Evolution and Marine Biology, University of California, Santa Barbara, Santa Barbara, CA 931069610, USA
7 Department of Biology, Pennsylvania State University, University Park, State College, PA 16802, USA

8 Department of Environmental Science and Policy, George Mason University, Fairfax, VA 22030-4444, USA

9 Department of Earth and Environmental Sciences, Paleontology and Geobiology, Ludwig-Maximilians-Universität München, Munich, Germany

10 GeoBio-Center, Ludwig-Maximilians-Universität München, Munich, Germany

11 Staatliche Naturwissenschaftliche Sammlungen Bayerns (SNSB)-Bayerische Staatssammlung für Paläontologie und Geologie, Munich, Germany

12 Department of Biology, Marine Biology \& Environmental Science, Roger Williams University, Bristol, RI 02809, USA 